\title{
Unfavourable effects of medically indicated oral anticoagulants on survival in idiopathic pulmonary fibrosis
}

\author{
Michael Kreuter ${ }^{1}$, Marlies S. Wijsenbeek ${ }^{2}$, Martina Vasakova ${ }^{3}$, Paolo Spagnolo ${ }^{4}$, \\ Martin Kolb ${ }^{5}$, Ulrich Costabel ${ }^{6}$, Derek Weycker ${ }^{7}$, Klaus-Uwe Kirchgaessler ${ }^{8}$ and \\ Toby M. Maher ${ }^{9}$
}

\begin{abstract}
Affiliations: ${ }^{1}$ Center for Interstitial and Rare Lung Diseases, Pneumology and Respiratory Critical Care Medicine, Thoraxklinik, University of Heidelberg, and Translational Lung Research Center Heidelberg (TLRCH), Heidelberg, Germany; Member of the German Center for Lung Research (DZL), Germany. ${ }^{2}$ Dept of Pulmonary Medicine, Erasmus Medical Center, University Hospital Rotterdam, Rotterdam, The Netherlands. ${ }^{3}$ Dept of Respiratory Medicine, Thomayer Hospital, Prague, Czech Republic. ${ }^{4}$ Medical University Clinic, Canton Hospital Baselland and University of Basel, Liestal, Switzerland. ${ }^{5}$ Firestone Institute for Respiratory Health, Dept of Medicine, Pathology and Molecular Medicine, McMaster University, Hamilton, ON, Canada. ${ }^{6}$ Interstitial and Rare Lung Disease Unit, Ruhrlandklinik, University Hospital, University of Duisburg-Essen, Essen, Germany. ${ }^{7}$ Policy Analysis Inc. (PAI), Brookline, MA, USA. ${ }^{8}$ F. Hoffmann-La Roche Ltd, Basel, Switzerland. ${ }^{9}$ National Institute for Health Research Biomedical Research Unit, Royal Brompton Hospital and Fibrosis Research Group, National Heart and Lung Institute, Imperial College, London, UK.
\end{abstract}

Correspondence: Michael Kreuter, Center for Interstitial and Rare Lung Diseases, Pneumology and Respiratory Critical Care Medicine, Thoraxklinik, Heidelberg University Hospital, Röntgenstrasse 1, 69120 Heidelberg, Germany. E-mail: kreuter@uni-heidelberg.de

ABSTRACT Procoagulant and antifibrinolytic activity has been associated with idiopathic pulmonary fibrosis (IPF); however, investigation of anticoagulant therapy in IPF has suggested deleterious effects. This post hoc analysis evaluated the effect of medically indicated anticoagulation on mortality and other clinical outcomes in IPF.

Patients randomised to placebo $(n=624)$ from three controlled trials in IPF were analysed by oral anticoagulant use. End-points included all-cause and IPF-related mortality, disease progression, hospitalisation, and adverse events, over 1 year.

At baseline, $32(5.1 \%)$ patients randomised to placebo were prescribed anticoagulants for non-IPF indications, 29 (90.6\%) of whom received warfarin. Unadjusted analyses demonstrated significantly higher allcause and IPF-related mortality at 1 year in baseline anticoagulant users versus nonusers (15.6\% versus $6.3 \%$, $\mathrm{p}=0.039$ and $15.6 \%$ versus $3.9 \%, \mathrm{p}=0.002$, respectively). In multivariate analyses, baseline use of anticoagulants was an independent predictor of IPF-related mortality (hazard ratio 4.7, $\mathrm{p}=0.034$ ), but not other end-points. Rates of bleeding and cardiac events did not differ significantly between groups. In an exploratory analysis, anticoagulant use at any time during the study was an independent predictor of all end-points.

This post hoc analysis suggests that anticoagulants used for non-IPF indications may have unfavourable effects in IPF patients. Future studies are needed to explore this relationship further.

@ERSpublications

Anticoagulant use for medically indicated comorbidities may have unfavourable effects in IPF http://ow.ly/XZC9j

This article has been revised according to the correction published in the August 2016 issue of the European Respiratory Journal.

This article has supplementary material available from erj.ersjournals.com

Received: Dec 102015 | Accepted after revision: Jan 252016 | First published online: April 212016

Support statement: The sponsor, F. Hoffmann-La Roche, funded the analysis of the data by Policy Analysis Inc. (PAI). Funding information for this article has been deposited with FundRef.

Conflict of interest: Disclosures can be found alongside the online version of this article at erj.ersjournals.com

Copyright OERS 2016 


\section{Introduction}

Idiopathic pulmonary fibrosis (IPF) is a chronic, progressive and irreversible lung disease with an estimated median survival of 2-5 years following diagnosis [1]. Lung function deteriorates over time causing disabling dyspnoea and eventually death, most commonly from respiratory failure [2].

The pathophysiology of IPF is not yet fully characterised, although evidence suggests that the coagulation cascade may be involved in lung inflammation and fibrosis [3]. In vitro studies have demonstrated higher levels of tissue factor, the key initiator of coagulation, in bronchoalveolar lavage fluid from patients with IPF compared with samples from patients without IPF [4]. This evidence suggested a link between fibroproliferative changes and activation of the coagulation cascade by profibrotic mediators, such as protease-activated receptor- 2 and factor X $[5,6]$. A recent case-control study of 211 patients with IPF found that they were over four times more likely to exhibit a prothrombotic state (defined as at least one inherited or acquired clotting defect) compared with population controls. In patients with IPF, a prothrombotic state was associated with a threefold increased risk of death [7]. These findings were supported by an analysis of mortality data from a US healthcare database of over 218000 patients with IPF and by a Danish registry study of nearly 20000 patients with idiopathic interstitial pneumonitis. Both studies found a higher prevalence of venous thromboembolism in patients with IPF compared with population controls [8, 9].

The potential link between the coagulation system and lung fibrosis has led to investigation of anticoagulants as a treatment for IPF. A pre-clinical study found that injection of heparin reduced collagen deposition in the lungs of bleomycin-treated mice [10], and another study demonstrated that direct inhibition of thrombin significantly reduced fibroblast proliferation, procollagen production and connective tissue growth factor mRNA expression in the lungs of bleomycin-treated rats [11]. Positive pre-clinical results led to human studies. A Japanese trial randomised 56 patients with IPF to receive prednisolone alone or in combination with warfarin. Patients treated with warfarin had a reduction in the secondary outcome of IPF acute exacerbation-associated mortality compared with those treated with prednisolone alone [12], although this trial was associated with significant methodological flaws [13]. Indeed, when warfarin was given as a treatment for IPF in the randomised, double-blind, placebo-controlled AntiCoagulant Effectiveness in Idiopathic Pulmonary Fibrosis (ACE-IPF) study, a significantly higher all-cause mortality rate was found in patients treated with warfarin versus placebo and the study was terminated early [14]. The summary of the evidence suggests that anticoagulants given specifically for the treatment of IPF may be associated with negative outcomes. Therefore, a strong recommendation against the use of anticoagulants in IPF has been given in the recent update of the IPF guidelines [13].

Despite this, recent registry data suggest that anticoagulants continue to be used in patients with IPF in clinical practice [15]; therefore, it is important to clearly understand the impact of this class of medications when taken by patients with IPF. The aim of this post hoc analysis was to investigate the effect of anticoagulants prescribed for non-IPF medical indications on mortality, disease progression and other outcomes in patients with IPF.

\section{Methods}

Patients

This post hoc analysis utilised data from patients randomised to the placebo arm of three phase III, controlled, double-blind trials of pirfenidone in IPF: Assessment of Pirfenidone to Confirm Efficacy and Safety in Idiopathic Pulmonary Fibrosis (ASCEND; study 016; NCT01366209) and Clinical Studies Assessing Pirfenidone in Idiopathic Pulmonary Fibrosis: Research of Efficacy and Safety Outcomes (CAPACITY; studies 004 and 006; NCT00287729 and NCT00287716) [16, 17]. Eligibility criteria for the ASCEND and CAPACITY studies have been described previously [16, 17]. In brief, patients were aged between 40 and 80 years with a diagnosis of IPF confirmed by high-resolution computed tomography (HRCT) alone or HRCT plus surgical lung biopsy, and forced vital capacity (FVC) $\%$ pred $\geqslant 50 \%$ (and $\leqslant 90 \%$ in ASCEND), diffusing capacity of the lung carbon monoxide (DLCO) $\%$ pred $\geqslant 35 \%(\geqslant 30 \%$ and $\leqslant 90 \%$ in ASCEND) and 6 -min walking distance $(6 \mathrm{MWD}) \geqslant 150 \mathrm{~m}$. Exclusion criteria included obstructive lung disease, clinically significant concomitant diseases, unstable IPF, active infection and malignancy [16, 17]. Both trials were conducted in accordance with the International Conference on Harmonisation Guidelines, the Declaration of Helsinki, and the relevant local legal and regulatory requirements of the countries the trials were conducted in. Written, informed consent was obtained from patients before screening and the study protocols were approved by the institutional review board or ethics committee at each participating centre.

\section{Study outcomes}

The effect of anticoagulant use on the following end-points during a 1-year follow-up period was investigated: all-cause mortality, IPF-related mortality, disease progression (defined as the first occurrence of any one of the following: $\geqslant 10 \%$ decline in FVC \% pred (confirmed at two consecutive assessments $\geqslant 6$ weeks 
apart), confirmed $\geqslant 50 \mathrm{~m}$ decline in $6 \mathrm{MWD}$, or death) changes from baseline in FVC and 6MWD, and all-cause hospitalisation. Adverse event data, particularly bleeding and cardiac events, were also analysed by anticoagulant use.

\section{Statistical analysis}

For analyses, patients were stratified into two subgroups based on use of anticoagulants at baseline (yes/no). Characteristics including lung function, medical history and cardiovascular risk factors were compared between baseline users and nonusers of anticoagulants using an independent samples t-test for continuous variables and a Chi-squared test for categorical variables.

The type of anticoagulant, the indication for use and the adverse events experienced during the studies were analysed descriptively. Unadjusted risks of binary study outcomes and changes from baseline in FVC and 6MWD were compared between baseline users and nonusers of anticoagulants using an independent samples t-test or a Chi-squared test. In addition, binary study outcomes were compared within a bivariate and multivariate framework, using Kaplan-Meier plots and Cox proportional hazards models. Patients were censored at the time of loss to follow-up, at the time of lung transplantation or at the end of the 1-year follow-up period, whichever occurred first. In multivariate models, the impact of anticoagulant use on study outcomes was adjusted for lung function, medical history and cardiovascular risk factors. The presence of multicollinearity, hazards assumptions and treatment of death as a competing risk were evaluated using published methods $[18,19]$.

An exploratory analysis was conducted to compare patients who were treated with anticoagulants at any time during the studies with patients who did not receive anticoagulants. The study end-points collected for these patients and the statistical methods used for analysis were the same as for the baseline-use analysis.

\section{Results}

Baseline use of anticoagulants

Patients

A total of 624 patients were included in the post hoc analyses, of whom 32 (5.1\%) were taking anticoagulants at baseline. The duration $($ mean \pm SD) of follow-up was $319.3 \pm 99.5$ days in baseline users of anticoagulants and $346.6 \pm 61.2$ days in nonusers.

Baseline users of anticoagulants were older than nonusers, with a mean age of 71.1 versus 66.9 years, respectively ( $p=0.0025)$. Baseline users of anticoagulants had a significantly lower mean 6MWD compared with nonusers (368.2 versus $414.1 \mathrm{~m}$, respectively, $\mathrm{p}=0.008$ ) (table 1 ). In addition, baseline users showed a significantly higher prevalence of cardiovascular disease, chronic renal failure, pulmonary embolism, pulmonary hypertension, atrial fibrillation and deep vein thrombosis versus baseline nonusers. No significant differences were noted in cardiovascular risk factors between the two groups (table 1).

Of the patients taking anticoagulants at baseline, most were taking the vitamin $\mathrm{K}$ antagonists warfarin $(90.6 \%)$ or acenocoumarol $(3.1 \%)$. Of the two patients taking an anticoagulant other than a vitamin $\mathrm{K}$ antagonist, both were taking dabigatran (online supplementary table S1). The most common indication for anticoagulant use was atrial fibrillation (34.4\%), followed by prophylaxis against thromboembolic events $(25.0 \%)$ and deep vein thrombosis (18.8\%) (online supplementary table S1).

\section{Disease end-points}

In unadjusted analyses of study outcomes at 1 year (table 2), a significantly higher proportion of patients treated with anticoagulants at baseline versus nonusers experienced all-cause mortality (15.6\% versus $6.3 \%$, respectively, $\mathrm{p}=0.039)$ and IPF-related mortality $(15.6 \%$ versus $3.9 \%, \mathrm{p}=0.0018)$ (figure 1$)$. The unadjusted 1 -year risk of disease progression did not differ between the two groups (table 2). No significant difference was observed between the two groups for change from baseline in FVC \% pred (figure 2), 6MWD or all-cause hospitalisation (table 2). In multivariate analyses, use of anticoagulants at baseline was not an independent predictor of any of the end-points tested except IPF-related mortality (hazard ratio 4.7, 95\% CI 1.1-19.3, p=0.034) (table 3).

For patients treated with anticoagulants at baseline, all deaths $(n=5)$ were attributed to IPF. In nonusers, 23 of 37 deaths were IPF-related (table 2). The time (mean \pm SD) to IPF-related mortality was $25.6 \pm 9.8$ weeks in baseline users of anticoagulants and $30.8 \pm 14.6$ weeks in baseline nonusers. An analysis of FVC decline from baseline to the visit prior to IPF-related death found that baseline users of anticoagulants showed a mean \pm SD decline in FVC from baseline of $4.2 \pm 5.96 \%$ versus a mean decline of $14.6 \pm 7.68 \%$ in baseline nonusers.

In addition, comparison of mortality rates in baseline vitamin $\mathrm{K}$ antagonist users versus baseline all-anticoagulant users showed little difference in mortality between the two groups $(16.7 \%$ versus $15.6 \%$, respectively, for both all-cause and IPF-related mortality). 
TABLE 1 Summary of baseline demographics: baseline anticoagulant users and nonusers

\begin{tabular}{|c|c|c|c|}
\hline & Anticoagulant users & Anticoagulant nonusers & p-value \\
\hline Subjects & 32 & 592 & \\
\hline Age years & $71.1 \pm 6.8$ & $66.9 \pm 7.5$ & 0.0025 \\
\hline Male & $24(75.0)$ & $441(74.5)$ & 0.9489 \\
\hline FVC \% pred & $70.0 \pm 11.7$ & $72.1 \pm 13.7$ & 0.3996 \\
\hline DLco $\%$ pred & $43.3 \pm 8.4$ & $45.7 \pm 11.2$ & 0.1290 \\
\hline 6MWD m & $368.2 \pm 109.4$ & $414.1 \pm 92.9$ & 0.0080 \\
\hline UCSD SOBQ score & $38.2 \pm 23.8$ & $34.7 \pm 21.5$ & 0.3697 \\
\hline \multicolumn{4}{|l|}{ Medical history } \\
\hline Cardiovascular disease & $14(43.8)$ & $153(25.8)$ & 0.0259 \\
\hline Chronic renal failure & $3(9.4)$ & $17(2.9)$ & 0.0419 \\
\hline COPD & $1(3.1)$ & $21(3.5)$ & 0.8996 \\
\hline Pulmonary embolism & $5(15.6)$ & $1(0.2)$ & $<0.0001$ \\
\hline Pulmonary hypertension & $3(9.4)$ & $16(2.7)$ & 0.0324 \\
\hline Atrial fibrillation & $15(46.9)$ & $14(2.4)$ & $<0.0001$ \\
\hline Deep vein thrombosis & $9(28.1)$ & $8(1.4)$ & $<0.0001$ \\
\hline \multicolumn{4}{|l|}{ Cardiovascular risk factors } \\
\hline Hypertension & $20(62.5)$ & $320(54.1)$ & 0.3500 \\
\hline Smoker current/ex & $22(68.8)$ & $362(61.1)$ & 0.3893 \\
\hline Diabetes & $8(25.0)$ & 126 (21.3) & 0.6180 \\
\hline Hypercholesterolaemia & $18(56.3)$ & $277(46.8)$ & 0.2965 \\
\hline Obese $\left(\mathrm{BMI}>30 \mathrm{~kg} \cdot \mathrm{m}^{-2}\right.$ ) & $17(53.1)$ & $248(41.9)$ & 0.2105 \\
\hline \multicolumn{4}{|c|}{$\begin{array}{l}\text { Data are presented as } \mathrm{n} \text {, mean } \pm \text { SD or } \mathrm{n}(\%) \text {, unless otherwise stated. FVC: forced vital capacity; } \\
\text { DLCO: diffusing capacity of the lung for carbon monoxide; 6MWD: 6-min walk distance; UCSD SOBQ: } \\
\text { University of California San Diego Shortness of Breath Questionnaire; COPD: chronic obstructive pulmonary } \\
\text { disease; BMI: body mass index. }\end{array}$} \\
\hline
\end{tabular}

\section{Adverse events}

The incidence of bleeding and cardiac events did not differ significantly between groups. Bleeding was reported in $3.1 \%$ of baseline anticoagulant users and $3.7 \%$ of baseline nonusers ( $p=0.8627$ ), and $6.3 \%$ of baseline users and $9.3 \%$ of baseline nonusers experienced a cardiac event over the 1-year period $(\mathrm{p}=0.5609)$. There were no significant differences in other adverse events (table 4$)$.

\section{TABLE 2 Unadjusted 1-year risk of study outcomes for baseline anticoagulant users and nonusers}

\begin{tabular}{|c|c|c|c|}
\hline & $\begin{array}{c}\text { Baseline anticoagulant } \\
\text { users }\end{array}$ & $\begin{array}{c}\text { Baseline anticoagulant } \\
\text { nonusers }\end{array}$ & p-value \\
\hline Subjects & 32 & 592 & \\
\hline All-cause mortality & $5(15.6)$ & $27(4.6)$ & 0.0057 \\
\hline FVC (absolute) decrease $\geqslant 10 \%$ & $3(9.4)$ & $61(10.3)$ & 0.8660 \\
\hline $6 M W D$ decrease $\geqslant 150 \mathrm{~m}$ & $6(18.8)$ & $143(24.2)$ & 0.4848 \\
\hline FVC \% pred change observed from baseline on the visit prior to death & $-4.2 \pm 5.96$ & $-14.6 \pm 7.68$ & \\
\hline Time to mortality" weeks & $25.6 \pm 9.78$ & $30.8 \pm 14.57$ & \\
\hline \multicolumn{4}{|l|}{ FVC change } \\
\hline Absolute decrease $\geqslant 10 \%$ & $6(18.8)$ & $107(18.1)$ & 0.9230 \\
\hline Relative decrease $\geqslant 10 \%$ & $11(34.4)$ & $174(29.4)$ & 0.5477 \\
\hline FVC \% pred change observed & $-6.0 \pm 6.4^{\S}$ & $-5.2 \pm 6.9^{f}$ & 0.5274 \\
\hline
\end{tabular}




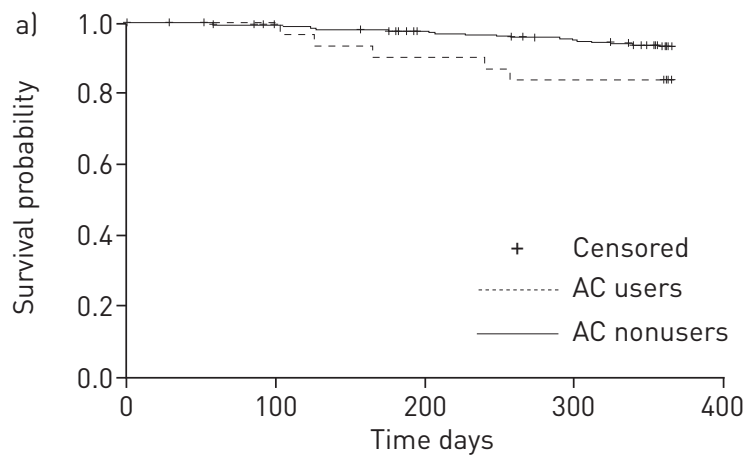

Patients at risk $n$

AC users 32

AC nonusers 592

$\begin{array}{cccc}31 & 28 & 26 & 0 \\ 582 & 561 & 544 & 0\end{array}$

\section{Patients at risk $n$}

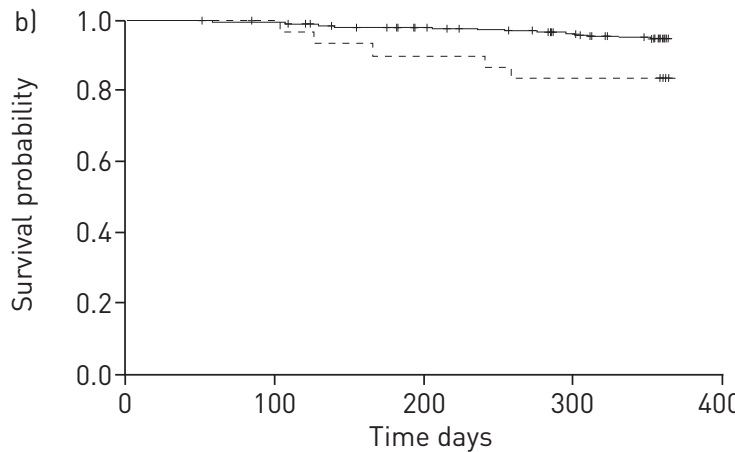

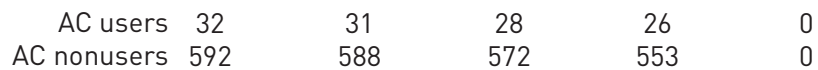

FIGURE 1 Unadjusted 1-year risk of a) all-cause and b) idiopathic pulmonary fibrosis-related mortality: baseline anticoagulant (AC) users and nonusers. $p$-values based on the log-rank test: a) $p=0.030$ and b) $p=0.0011$.

\section{Any use of anticoagulants}

Patients

A total of 22 patients started treatment with anticoagulants during the trial, resulting in 54 ever-users of anticoagulants. Baseline demographics suggested that anticoagulant ever-users were older (mean age 70.7 versus 66.8 years, respectively, $\mathrm{p}=0.0002)$, and had significantly lower mean DLCO \% pred $(42.6 \%$ versus $45.9 \%$, respectively, $\mathrm{p}=0.0091$ ) and $6 \mathrm{MWD}(374.2$ versus $415.4 \mathrm{~m}$, respectively, $\mathrm{p}=0.0023$ ) compared with nonusers (online supplementary table S2). A comparison of comorbidities at baseline also highlighted a significantly higher prevalence of cardiovascular disease, chronic renal failure, pulmonary embolism, atrial fibrillation and deep vein thrombosis in ever-users compared with nonusers. Cardiovascular risk factors did not differ significantly between groups (online supplementary table S2).

Most anticoagulant ever-users were taking warfarin $(81.5 \%)$, with the remainder receiving dabigatran (7.4\%), fondaparinux (7.4\%), bivalirudin $(1.9 \%)$ or acenocoumarol $(1.9 \%)$. The most common indication

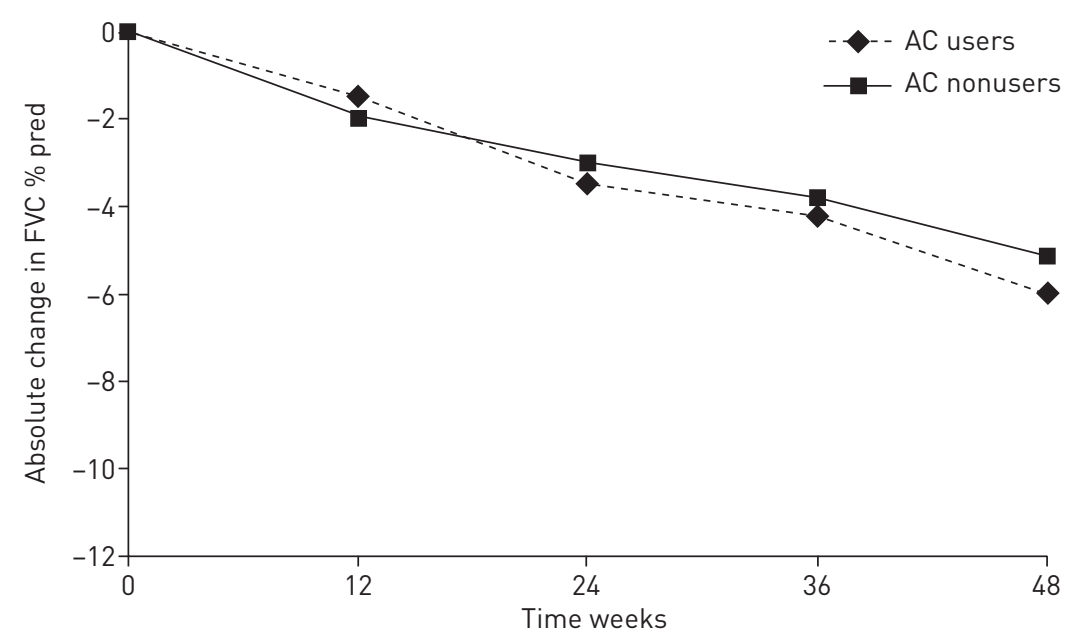

Patients $\mathrm{n}$

$\begin{array}{lccccc}\text { Baseline AC users } & 32 & 31 & 28 & 25 & 26 \\ \text { Baseline AC nonusers } & 592 & 573 & 563 & 535 & 523 \\ \text { FVC \% pred change } & & & & & -6.0 \\ \text { Baseline AC users } & 0.0 & -1.5 & -3.5 & -4.2 & -5.2 \\ \text { Baseline AC nonusers } & 0.0 & -1.9 & -3.0 & -3.8 & -0.9 \\ \text { Absolute difference } & - & 0.4 & -0.5 & -0.3 & 0.527 \\ \text { p-value } & - & 0.628 & 0.671 & 0.803 & \end{array}$

FIGURE 2 Absolute observed change in forced vital capacity (FVC) \% pred: baseline anticoagulant (AC) users and nonusers. 
TABLE 3 Bivariate and multivariate analyses: baseline anticoagulant users versus nonusers

\begin{tabular}{|c|c|c|c|c|}
\hline & \multicolumn{2}{|c|}{ Bivariate analyses } & \multicolumn{2}{|c|}{ Multivariate analyses } \\
\hline & $\begin{array}{l}\text { Hazard ratio } \\
(95 \% \mathrm{CI})\end{array}$ & p-value & $\begin{array}{l}\text { Hazard ratio } \\
\text { (95\% CI) }\end{array}$ & p-value \\
\hline $\begin{array}{l}\text { Disease progression }{ }^{\#} \\
\text { Mortality }\end{array}$ & $1.2(0.7-2.1)$ & 0.435 & $1.2(0.6-2.6)$ & 0.600 \\
\hline All-cause mortality & $2.7(1.1-6.9)$ & 0.037 & $2.6(0.7-8.8)$ & 0.136 \\
\hline IPF-related mortality & $4.4(1.7-11.5)$ & 0.003 & $4.7(1.1-19.3)$ & 0.034 \\
\hline \multicolumn{5}{|l|}{ FVC change } \\
\hline Absolute decrease $\geqslant 5 \%$ & $1.3(0.8-2.2)$ & 0.284 & $1.2(0.6-2.6)$ & 0.578 \\
\hline Relative decrease $\geqslant 5 \%$ & $1.1(0.7-1.8)$ & 0.773 & $1.0(0.5-1.9)$ & 0.936 \\
\hline Absolute decrease $\geqslant 10 \%$ & $1.2(0.5-2.6)$ & 0.736 & $1.6(0.5-5.3)$ & 0.438 \\
\hline Relative decrease $\geqslant 10 \%$ & $1.3(0.7-2.4)$ & 0.370 & $1.2(0.5-2.9)$ & 0.743 \\
\hline Death or FVC (absolute) $\geqslant 10 \%$ decrease & $1.6(1.0-2.8)$ & 0.065 & $1.5(0.7-3.3)$ & 0.316 \\
\hline Death or $6 M W D$ decrease $\geqslant 50 \mathrm{~m}$ & $1.2(0.7-2.1)$ & 0.528 & $1.2(0.5-2.5)$ & 0.701 \\
\hline All-cause hospitalisation & $1.2(0.6-2.7)$ & 0.581 & $1.2(0.4-3.2)$ & 0.766 \\
\hline
\end{tabular}

IPF: idiopathic pulmonary fibrosis; FVC: forced vital capacity; 6MWD: 6-min walk distance. " : defined as $\geqslant 10 \%$ decrease in FVC \% pred, $\geqslant 50 \mathrm{~m}$ decline in $6 \mathrm{MWD}$ or death.

for use was atrial fibrillation (33.3\%), followed by prophylaxis (22.2\%) and deep vein thrombosis (20.4\%) (online supplementary table S1).

\section{Disease end-points}

In unadjusted analyses, a significantly greater proportion of anticoagulant ever-users versus nonusers experienced disease progression (57.4\% versus $37.9 \%, \mathrm{p}=0.0097)$, all-cause mortality $(20.4 \%$ versus $5.4 \%$, respectively, $\mathrm{p}<0.0001)$, IPF-related mortality $(18.5 \%$ versus $3.2 \%, \mathrm{p}<0.0001)$ and all-cause hospitalisation ( $38.9 \%$ versus $17.2 \%, \mathrm{p}=0.0001$ ) (online supplementary table $\mathrm{S} 3$ ). In multivariate analyses, any use of anticoagulants during the study was an independent predictor of all end-points tested (online supplementary table S4).

TABLE 4 Adverse events in baseline anticoagulant users and nonusers

Baseline anticoagulant

Subjects
Adverse events affecting $\geqslant 10 \%$ of baseline
anticoagulant users
Cough
Upper respiratory tract infection
Diarrhoea
IPF
Nausea
Dyspnoea
Fatigue
Headache
Nasopharyngitis
Bronchitis
Peripheral oedema
Back pain
Constipation
Dizziness
Rash
Sinusitis
Additional adverse events of interest
Cardiac events
Bleeding
users

32

Baseline anticoagulant nonusers

592

$\begin{array}{lc}6(18.8) & 178(30.1) \\ 6(18.8) & 153(25.8) \\ 8(25.0) & 121(20.4) \\ 8(25.0) & 120(20.3) \\ 7(21.9) & 91(15.4) \\ 3(9.4) & 127(21.5) \\ 2(6.3) & 117(19.8) \\ 4(12.5) & 116(19.6) \\ 5(15.6) & 110(18.6) \\ 5(15.6) & 92(15.5) \\ 5(15.6) & 50(8.4) \\ 4(12.5) & 62(10.5) \\ 4(12.5) & 69(11.7) \\ 3(9.4) & 68(11.5) \\ 1(3.1) & 65(11.0) \\ 2(6.3) & 63(10.6) \\ 2(6.3) & 55(9.3) \\ 1(3.1) & 22(3.7)\end{array}$

Data are presented as $\mathrm{n}$ or $\mathrm{n}(\%)$. IPF: idiopathic pulmonary fibrosis. 
Adverse events

Ever-users of anticoagulants had a significantly higher incidence of cardiac events compared with nonusers of anticoagulants ( $18.5 \%$ versus $8.2 \%$, respectively, $\mathrm{p}=0.0123)$; however, there was no difference in bleeding events, with an incidence of $3.7 \%$ reported in both groups.

\section{Discussion}

In this post hoc analysis, patients in the placebo groups treated with anticoagulants at baseline in the CAPACITY and ASCEND trials had a higher rate of all-cause and IPF-related mortality compared with baseline nonusers. Although only a small number of patients received anticoagulants in CAPACITY and ASCEND, a recent German IPF registry found that $20 \%$ of patients were receiving anticoagulants [15]. Therefore, it is possible that a large number of patients with IPF could be affected by an unfavourable effect of anticoagulants and it is important to understand this finding further.

The increase in IPF-related mortality plus a trend for a higher rate of hospitalisation, and the observation that all deaths in anticoagulant users were attributed to IPF, suggests that anticoagulants may contribute to an underlying worsening of respiratory status in patients with IPF. This is supported by the ACE-IPF trial, which also observed a deleterious effect of anticoagulation on mortality and IPF exacerbations [14]. The increase in mortality observed in our analysis, and that observed in the ACE-IPF trial [14], could not be explained by an increased rate of clinical bleeding leading to death. However, it is feasible that the underlying mechanism for the effect of anticoagulation on mortality in IPF is related to an increase in subclinical pulmonary bleeding, leading to an aggravation of pulmonary fibrosis, including a decline in respiratory function and ultimately death [20].

Many patients with IPF require anticoagulants for comorbid indications and, therefore, further research is needed to elucidate the safest way of treating these patients. One suggestion is that the detrimental effect observed in our analysis and other studies may be limited to vitamin $\mathrm{K}$ antagonists alone [21]. The small number of patients receiving other anticoagulants in the CAPACITY and ASCEND trials prevented investigation of a drug-specific effect in our analysis. However, vitamin $\mathrm{K}$ antagonists affect multiple coagulation factors, noncoagulant vitamin-K-dependent proteins and levels of protein $\mathrm{C}$ (and protein S) [3, 21, 22]. While our results raise some questions over the role of a procoagulant state in the pathogenesis of IPF, they may also be explained by the nonselective mode of action of vitamin $\mathrm{K}$ antagonist. It is possible that newer, more specific anticoagulants, such as direct thrombin inhibitors and direct factor $\mathrm{X}$ inhibitors [22], may not increase mortality in IPF [21], but further research is required to determine whether this is the case and to elucidate the molecular mechanisms of the effect of anticoagulation (or vitamin $\mathrm{K}$ antagonists) on IPF mortality. In addition, future research should explore novel anticoagulation approaches in IPF, such as modulating the signalling pathways that control coagulation responses $[23,24]$.

Other studies that have investigated the use of vitamin $\mathrm{K}$ antagonists in IPF for non-IPF medical indications support the findings of our analysis, including a retrospective study in which the 16 patients receiving vitamin $\mathrm{K}$ antagonists had a significantly higher risk of mortality compared with the 105 patients not receiving anticoagulants [25]. Similarly, another retrospective study found that patients with IPF treated with warfarin had poorer 1- and 3-year survival and a shorter time to disease progression compared with patients who did not receive anticoagulants [26]. Case studies also suggest that warfarin may induce acute exacerbations of IPF [21].

Conversely, the results of a Japanese trial of prednisolone with or without warfarin found a reduction in the secondary outcome of IPF acute exacerbation-associated mortality in patients receiving warfarin [12]. However, this trial had several methodological weaknesses, including a lack of information regarding how patients were randomised to treatment, absence of blinding and ambiguity surrounding how discontinuation from the study was handled. Furthermore, there was a high withdrawal rate in the anticoagulant treatment arm, a high incidence of acute exacerbations of IPF and a failure to exclude pulmonary embolism as a potential cause of acute deterioration. Therefore, this trial was discounted from a recent update to IPF treatment guidelines, which strongly recommended against use of warfarin in the treatment of IPF [13].

It should be acknowledged that the underlying comorbidities for which anticoagulants were indicated in our analysis could have contributed to the observed increase in mortality even though all deaths were attributed to IPF. Anticoagulant users were also older and had a higher prevalence of comorbidities overall compared with nonusers. This could explain why only the unadjusted analyses, with the exception of IPF-related mortality $(\mathrm{p}=0.034)$, were of statistical significance and why nonusers of anticoagulants had a greater FVC decline between baseline and the last visit prior to death than users of anticoagulants. IPF-related mortality was statistically significant in the multivariate analysis of baseline anticoagulant use, suggesting a negative influence of anticoagulation on IPF. Although all deaths in users of anticoagulants at baseline were recorded as being due to IPF, the number of deaths was small and the underlying cause of 
death may have been affected by the comorbid condition. However, while results of the analysis of any anticoagulant use should also be interpreted with caution, the significant effect of any anticoagulant use on IPF-related mortality and FVC cannot be explained only by the indicating comorbid conditions.

In addition to the potential bias of comorbid conditions in patients who received anticoagulants, this analysis was limited by its post hoc methodology, the lack of international normalised ratio values for patients treated with warfarin, and the small number of patients treated with anticoagulants in the placebo arms of the ASCEND and CAPACITY trials. Furthermore, patients with advanced IPF and more significant cardiovascular and thromboembolic disease were excluded from these trials $[16,17]$. However, these results provide useful insight to the potentially unfavourable effects of medically indicated anticoagulants when used in IPF. Future studies should investigate further whether the unfavourable effect noted in this analysis is attributable to the mechanism of action of anticoagulants or their indication for use. In addition, as our results may be mainly related to the use of vitamin $\mathrm{K}$ antagonists, it is necessary to explore the possibility of a class effect with vitamin $\mathrm{K}$ antagonists versus other classes of anticoagulants to determine if there are alternative medications that may provide a better risk profile for prescribing anticoagulation in IPF.

In conclusion, the findings of our analysis suggest that anticoagulant use for medically indicated comorbidities may have unfavourable effects in IPF. Therefore, the use of anticoagulation in patients with IPF should be based on an individual assessment of the benefits and risks, and patients should be closely monitored during treatment.

\section{Acknowledgements}

Programming support was provided by Mark Atwood of Policy Analysis Inc. (PAI) (Brookline, MA, USA). Medical writing support was provided by Catherine Stanton on behalf of Complete Medical Communications Ltd (Macclesfield, UK), funded by F. Hoffmann-La Roche Ltd.

\section{References}

Meltzer EB, Noble PW. Idiopathic pulmonary fibrosis. Orphanet J Rare Dis 2008; 3: 8.

2 Ley B, Collard HR, King TE Jr. Clinical course and prediction of survival in idiopathic pulmonary fibrosis. Am J Respir Crit Care Med 2011; 183: 431-440.

3 Chambers RC. Procoagulant signalling mechanisms in lung inflammation and fibrosis: novel opportunities for pharmacological intervention? Br J Pharmacol 2008; 153: Suppl. 1, S367-S378.

4 Kotani I, Sato A, Hayakawa $\mathrm{H}$, et al. Increased procoagulant and antifibrinolytic activities in the lungs with idiopathic pulmonary fibrosis. Thromb Res 1995; 77: 493-504.

5 Scotton CJ, Krupiczojc MA, Königshoff M, et al. Increased local expression of coagulation factor X contributes to the fibrotic response in human and murine lung injury. J Clin Invest 2009; 119: 2550-2563.

6 Wygrecka M, Kwapiszewska G, Jablonska E, et al. Role of protease-activated receptor-2 in idiopathic pulmonary fibrosis. Am J Respir Crit Care Med 2011; 183: 1703-1714.

7 Navaratnam V, Fogarty AW, McKeever T, et al. Presence of a prothrombotic state in people with idiopathic pulmonary fibrosis: a population-based case-control study. Thorax 2014; 69: 207-215.

8 Sprunger DB, Olson AL, Huie TJ, et al. Pulmonary fibrosis is associated with an elevated risk of thromboembolic disease. Eur Respir J 2012; 39: 125-132.

9 Sode BF, Dahl M, Nielsen SF, et al. Venous thromboembolism and risk of idiopathic interstitial pneumonia: a nationwide study. Am J Respir Crit Care Med 2010; 181: 1085-1092.

10 Piguet PF, Van GY, Guo J. Heparin attenuates bleomycin but not silica-induced pulmonary fibrosis in mice: possible relationship with involvement of myofibroblasts in bleomycin, and fibroblasts in silica-induced fibrosis. Int J Exp Pathol 1996; 77: 155-161.

11 Howell DC, Goldsack NR, Marshall RP, et al. Direct thrombin inhibition reduces lung collagen, accumulation, and connective tissue growth factor mRNA levels in bleomycin-induced pulmonary fibrosis. Am J Pathol 2001; 159: 1383-1395.

12 Kubo H, Nakayama K, Yanai M, et al. Anticoagulant therapy for idiopathic pulmonary fibrosis. Chest 2005; 128: $1475-1482$.

13 Raghu G, Rochwerg B, Zhang Y, et al. An official ATS/ERS/JRS/ALAT clinical practice guideline: treatment of idiopathic pulmonary fibrosis. Am J Respir Crit Care Med 2015; 192: e3-e19.

14 Noth I, Anstrom KJ, Calvert SB, et al. A placebo-controlled randomized trial of warfarin in idiopathic pulmonary fibrosis. Am J Respir Crit Care Med 2012; 186: 88-95.

15 Behr J, Kreuter M, Hoeper MM, et al. Management of patients with idiopathic pulmonary fibrosis in clinical practice: the INSIGHTS-IPF registry. Eur Respir J 2015; 46: 186-196.

16 King TE Jr, Bradford WZ, Castro-Bernardini S, et al. A phase 3 trial of pirfenidone in patients with idiopathic pulmonary fibrosis. N Engl J Med 2014; 370: 2083-2092.

17 Noble PW, Albera C, Bradford WZ, et al. Pirfenidone in patients with idiopathic pulmonary fibrosis (CAPACITY): two randomised trials. Lancet 2011; 377: 1760-1769.

18 Allison PD. Survival Analysis Using SAS: A Practical Guide. 2nd Edn. Cary, SAS Institute, 2010.

19 Pencina MJ, D'Agostino RB Sr, D'Agostino RB Jr, et al. Evaluating the added predictive ability of a new marker: from area under the ROC curve to reclassification and beyond. Stat Med 2008; 27: 157-172.

20 Wijnen PA, Verschakelen JA, Bast A, et al. Diffuse alveolar hemorrhage in coumarin users: a fibrosing interstitial pneumonia trigger? Lung 2013; 191: 53-59.

21 Alagha K, Secq V, Pahus L, et al. We should prohibit warfarin in idiopathic pulmonary fibrosis. Am J Respir Crit Care Med 2015; 191: 958-960. 
22 Salem JE, Sabouret P, Funck-Brentano C, et al. Pharmacology and mechanisms of action of new oral anticoagulants. Fundam Clin Pharmacol 2015; 29: 10-20.

23 José RJ, Williams AE, Chambers RC. Proteinase-activated receptors in fibroproliferative lung disease. Thorax 2014; 69: 190-192.

24 Kataoka K, Taniguchi H, Kondoh Y, et al. Recombinant human thrombomodulin in acute exacerbation of idiopathic pulmonary fibrosis. Chest 2015; 148: 436-443.

25 Hyldgaard C, Hilberg O, Muller A, et al. A cohort study of interstitial lung diseases in central Denmark. Respir Med 2014; 108: 793-799.

26 Tomassetti S, Ruy JH, Gurioli C. The effect of anticoagulant therapy for idiopathic pulmonary fibrosis in real life practice. Sarcoidosis Vasc Diffuse Lung Dis 2013; 30: 121-127. 